\title{
GCU
}

Glasgow Caledonian

University

University for the Common Good

\section{Visual problems after stroke: a survey of current practice by occupational therapists working in UK stroke inpatient settings}

Pollock, Alex; Hazelton, Christine; Brady, Marian

Published in:

Topics in Stroke Rehabilitation

DOI:

$10.1310 /$ tsr18s01-643

Publication date:

2011

Document Version

Author accepted manuscript

Link to publication in ResearchOnline

Citation for published version (Harvard):

Pollock, A, Hazelton, C \& Brady, M 2011, 'Visual problems after stroke: a survey of current practice by

occupational therapists working in UK stroke inpatient settings', Topics in Stroke Rehabilitation, vol. 18, no. S1, pp. 643-651. https://doi.org/10.1310/tsr18s01-643

\section{General rights}

Copyright and moral rights for the publications made accessible in the public portal are retained by the authors and/or other copyright owners and it is a condition of accessing publications that users recognise and abide by the legal requirements associated with these rights.

Take down policy

If you believe that this document breaches copyright please view our takedown policy at https://edshare.gcu.ac.uk/id/eprint/5179 for details

of how to contact us. 
Visual problems after stroke: a survey of current practice by Occupational Therapists working in UK stroke inpatient settings. 


\section{Introduction}

Stroke is the greatest cause of severe disability in Scotland and the third most common cause of death (1). Within Scotland 13,000 people suffer from a new stroke each year (1) and half of all survivors are left with severe functional problems (2). There are many visual problems associated with stroke, including visual field deficits, disorders of eye movement and visuospatial neglect. The reported prevalence of these problems varies considerably. One recent multicentre prospective cohort study (3) found that a simple screening procedure was successful in identifying patients with visual difficulties. Of the 297 patients identified as having visual difficulties, 26\% had low visual acuity, 35\% had ocular pathologies, $68 \%$ had eye movement deficits, $49 \%$ had visual field impairments, $20 \%$ had perceptual deficits and $55 \%$ had a combination of 2 or more visual impairments.

Visual problems can impact on functional ability, quality of life, participation in rehabilitation and discharge destination following stroke (4-7). Furthermore, given that the incidence of stroke increases with age, a significant proportion of stroke patients have concurrent age-related visual problems (8) which, untreated, can adversely affect quality of life (9). Many age-related visual acuity problems can be effectively corrected with glasses, however there is evidence that often clinical staff do not ensure that stroke in-patients wear their prescribed glasses (10).

The evidence in relation to the effectiveness of management strategies for visual problems in stroke settings is limited (11). Consensus expert opinion within UK National Guidelines recommends that stroke patients should have assessments which evaluate visual acuity, visual field, eye movement and visuospatial neglect, and appropriate referral to and treatment from vision experts $(12,13)$. There are a few guidelines specifically relating to individual treatments for visual field deficits 
(13), but none of the UK guidelines make any specific recommendations about the types of treatments for patients with eye movement disorders.

It is widely acknowledged that the presence and impact of visual problems after stroke should be considered by all members of the multidisciplinary team (14), and that appropriate referral to vision experts should follow (13). Although orthoptists and ophthalmologists are experts in vision these professionals are generally not core members of the multidisciplinary team in stroke in-patient settings $(9,15)$. Within the core stroke multidisciplinary team several different health professionals, such as doctors, nurses, occupational therapists and physiotherapists, may consider and assess visual problems. However occupational therapists consider the initial assessment of visual problems, subsequent treatment and referral as a core part of their role $(16,17)$. Subsequently the occupational therapist is often the member of the multidisciplinary team with responsibility for the assessment and treatment and/or referral of patients with visual problems after stroke (although there may be some local variations in clinical practice). To our knowledge there is no documented evidence relating to the assessments, treatments and/or referrals made by occupational therapists relating to visual problems working in stroke in-patient settings in the UK. As part of a larger study to identify and document current clinical practice in Scotland for stroke patients with visual problems, we therefore felt it was important to investigate current practice in relation to the assessment and management of visual problems after stroke, as reported by occupational therapists (OTs) in Scottish stroke in-patient settings.

The primary aim of this study was therefore to explore, specific to visual problems after stroke, current practice, including assessments/protocols, referrals and 
treatments, of OTs working in Scottish stroke in-patient settings. Secondary aims were to explore whether we could identify particular training needs of OTs in relation to visual problems after stroke; to identify barriers to the management of visual problems; priorities for future stroke research in this field; and to explore whether there were any differences in the practice of stroke specialist OTs and non-specialist OTs.

\section{Methods}

\section{Study design}

A postal survey was chosen as the most effective and efficient method to study national visual assessment and management practices in geographically diverse units (18). Our methods adopted procedures which have been demonstrated to maximise response rates (18). The procedures included limiting the questionnaire to two-pages in length (one double-sided A4 page); using a university logo on all correspondence; using brown envelopes, with hand written addresses; sending letters by first class post, and using stamps (not franked); including stamped addressed return envelope with all correspondence; primarily asking fact-based questions, answered by ticking a box, and placing any more general, open, questions at the end of the questionnaire; avoiding sensitive questions and ensuring anonymity.

\section{Development of questionnaire}

Building on previous successful work in this area $(19,20)$ we designed a questionnaire to gather the required information from OTs. The questionnaire was designed to gather information about the stroke in-patient setting which the respondent worked in, vision assessments and protocols used, treatments 
administered and referrals made, and barriers experienced. For questions relating to treatment administered and referrals made the questionnaire was designed to gather information about treatments and referrals of patients with different visual problems. The categories of visual problems were designed to encompass the most common visual problems in the population of patients with stroke: visual field problem, eye movement problem, visual neglect, problems relating to spectacle use, and other visual problems.

Following initial development of the questionnaire, it was sent out to all members of the Scottish Stroke and Vision Steering Group and to all member of a multidisciplinary group which was formed to advise on a series of ongoing Cochrane systematic reviews relating to visual problems after stroke (21-23). Membership of these groups included health professionals, researchers, stroke survivors and representatives from Scottish charities relevant to stroke and vision. Recipients were asked to comment and provide feedback on all aspects of the questionnaire, including content, layout and comprehension. Twelve responses were received which primarily provided positive support for the questionnaire; a small number of very minor modifications were made to layout and structure following this feedback. The final questionnaire which was administered is in Appendix 1, and includes sections on 1) Care Setting, 2) Vision assessment / protocols, 3) Treatment of visual problems by OTs, 4) Barriers and support.

\section{Process}

The methodological process used is illustrated in Figure 1. Key stages involved the identification and agreement to participate (by telephone) of a named OT from each stroke in-patient setting in Scotland; introductory letter; first questionnaire (sent 1-3 weeks after introductory letter); second copy of questionnaire to non-responders 
(sent 2 weeks after first questionnaire). At the end of this process the unique identifiers for all OTs were destroyed; data from completed questionnaires were anonymously entered in an Excel spreadsheet, and analyses were completed using descriptive statistics.

\section{[FIGURE 1]}

\section{$\underline{\text { Results }}$}

A total of 61 stroke in-patient settings were identified in Scotland. One OT from each of these 61 settings agreed to receive a questionnaire. A total of 55 questionnaires (90\% response rate) were returned (32 after first mailing, and 23 after second mailing)(see figure 1).

\section{Care setting and Protocols}

Twenty three respondents (42\%) reported that they worked in a rehabilitation stroke in-patient setting, 12 (22\%) in an acute setting, and 20 (36\%) in a combined setting. Only $9 \%$ of respondents $(5 / 55)$ reported that their unit had a protocol or management plan for visual problems after stroke. Sixty-two percent of respondents (34/55) reported that their unit did not have a vision-related protocol or management plan, and 29\% (16/55) either did not know or did not answer this question.

\section{Vision Assessment}

Most respondents $(38 / 55 ; 69 \%)$ reported that they usually carried out visual assessment during their first OT assessment; although 16\% (9/55) reported that they carried out visual assessment if they thought the patient may have a visual problem. Only 4\% (2/55) reported that they carried out visual assessment if another member of the multidisciplinary team thought it was necessary. Two thirds of respondents $(37 / 55 ; 67 \%)$ reported that on average patients were less than 2 weeks after stroke 
at the time of assessment; with 18\% (10/55) reporting that patients were 2-6 weeks after stroke.

Eighty-two percent of respondents (45/55) reported that it was routine practice to ensure that patients had their prescribed glasses on the ward; $11 \%(6 / 55)$ reported that it was not routine practice and 7\% (4/55) did not know or did not respond.

Table 1 presents the reported frequency of using a variety of visual assessments and tests. The majority of respondents (49/55; 89\%) reported that they would assess visual attention and visual scanning with every patient or regularly. Over half of the OTs reported that they would use assessment of visual fields (confrontation)(37/55; 67\%), visual tracking(36/55; 65\%), visual memory (31/55; $56 \%)$, pattern recognition $(29 / 55 ; 53 \%)$ and use the Behavioural Inattention Test (28/55; 51\%) with every patient or regularly. In contrast, between one-third and one-half of OTs report that would rarely or never assess visual acuity (18/55 to $23 / 55$; 33-42\%), binocular function (23/55; $42 \%)$, or ocular movements $(27 / 55 ; 49 \%)$; and over half of OTs report that they would rarely or never assess contrast sensitivity, or use the Biometrics e-link Evaluation (32/55; 58\%), Brain Injury Visual Assessment Battery (34/55; 62\%) or Motor Free Visual Perception Test (35/55; $64 \%)$.

\section{[TABLE 1]}

\section{Treatment of visual problems by OTs}

\section{General management and referral}

Table 2 shows the reported frequency of 'likely outcomes' in patients found to have a visual problem. Approximately half of OTs reported that they would refer patients with visual field problems $(25 / 55 ; 45 \%)$ and eye movement problems $(29 / 55 ; 53 \%)$ to 
an ophthalmologist; while only a quarter of OTs report referring patients with these problems to an orthoptist (14/55 and 13/55; 25 and 24\% respectively). Very few patients are reported to be referred to hospital or community low vision service or psychologist. Over $82 \%(45 / 55)$ of OTs report delivering treatment to patients with visual neglect, and 69\% (38/55) for visual field problems. In contrast only $11 \%(6 / 55)$ of OTs report delivering treatment to patients with eye movement problems. Seventeen percent of respondents (9/55) did not answer the question on management of eye movement disorders, with one respondent commenting that they had "never experienced" a patient with such a disorder.

\section{[TABLE 2]}

\section{Specific treatment interventions}

Table 3 shows the number of OTs reporting use of specific treatments for patients with the different categories of visual problems after stroke, and also shows, as a percentage of total reported treatments used, the relative use of different treatments for each visual problem. It is notable that the percentage of respondents reporting use of specific interventions for visual field problems, eye movement problems and visual neglect were very similar for all interventions.

$75 \%$ of respondents $(41 / 55)$ reported that they would treat patients within 6 weeks of stroke (45\% (25/55) reported starting treatment less than 2 weeks after stroke; 30\% (16/55) reported starting treatment between 2 and 6 weeks after stroke). Most respondents $(21 / 55 ; 38 \%)$ reported that they generally provided treatment for up to up to 3 months.

[TABLE 3]

\section{Barriers and support}




\section{Barriers}

Figure 2 shows the reported barriers to the management of visual problems after stroke. The three main barriers identified by the respondents were the lack of a visual care protocol or management plan (by 33/55; 60\%); lack of specialist training (by $27 / 55 ; 49 \%$ ) and lack of awareness of the best treatment options (25/55; 45\%).

\section{Training}

When questioned about the training they had received specific to visual problems, a quarter of respondents $(13 / 55 ; 24 \%)$ reported that they had received no training specific to visual problems after stroke. Respondents described receiving training relating to general assessment $(34 / 55 ; 62 \%)$, general management $(24 / 55 ; 44 \%)$, aetiology (17/55; 31\%), specific tests / batteries (16/55; 29\%) and specific management $(6 / 55 ; 11 \%)$ of visual problems.

\section{Priorities}

Only $60 \%(33 / 55)$ of OTs responded to the section on priorities for future research (which required the entry of free text, rather than ticking a box). Most of these respondents $(23 / 33 ; 70 \%)$ identified priorities relating to evidence to support management \& treatment of visual problems following stroke. Other identified research priorities related to identification of effective assessments (10/33; 30\%), protocols for visual management $(6 / 33 ; 18 \%)$, and referral pathways $(2 / 33 ; 6 \%)$.

\section{[FIGURE 2]}

\section{Differences between specialists and non-specialists}

We explored the differences in responses between "stroke specialist OTs" and "non specialist OTs". Thirty-two responders (58\%) classed themselves as a stroke 
specialist OT, and 23 responders (42\%) as a non-specialist. A greater proportion of stroke specialist OTs than non-specialists reported using specific assessments of vision regularly or with every patient: greatest differences existed in the use of assessments of visual memory (23/32 (72\%) of specialist OTs and 9/ $23(40 \%)$ of non-specialists); visual tracking (25/32 (78\%) and 10/23 (45\%) respectively); binocular function (13/32 (41\%) and 2/23 (10\%) respectively) and visual scanning (31/32 (97\%) and 17/23 (75\%) respectively).

\section{Discussion}

We have obtained comprehensive reports from OTs from Scottish stroke inpatient settings relating to the management of visual problems after stroke. We have gathered evidence that OTs play a key role in the assessment and management of visual problems within Scottish stroke inpatient settings. This questionnaire received a very high response rate, demonstrating the effectiveness of the strategies adopted to increase response. We have therefore been successful in gaining comprehensive information reflective of current practice specific to visual problem after stroke by OTs in Scottish stroke inpatient settings. Only $9 \%$ of respondents reported that their stroke inpatient setting used a protocol for the assessment and management of visual problems. The absence of protocols in the majority of Scottish stroke inpatient settings is reflective of the low priority accorded to this group of problems experienced by patients following stroke. It is also likely to result in inconsistencies in the management of visual problems after stroke, both within and between stroke inpatient settings. OTs appear to be aware of the need for a visual care protocol as the absence of a visual care protocol or management plan was the most commonly reported barrier to the management of visual problems after stroke. The introduction of protocols or management plans for visual problems, across all Scottish stroke inpatient settings, therefore arguably ought to be a priority. 
Despite the absence of protocols in the majority of Scottish stroke inpatient settings, it is clear that OTs have a key role in the assessment and management of visual problems within Scottish in-patient stroke inpatient settings. All respondents reported some involvement in the assessment, management or referral of visual problems, with $69 \%$ of OTs assessing vision as part of their initial OT assessment. The high proportion (82\%) of stroke inpatient settings reporting that they ensure patients had their prescribed glasses on the ward is encouraging, as this is in contrast to previous research (10) which has suggested that many patients do not have their prescribed glasses. This may be reflective of improved practice over time, or may relate to geographical differences.

The majority of OTs (89\%) reported that they would routinely assess visual scanning and visual attention; these tests are associated with visual neglect and these results suggest OTs take a very active interest in visual neglect after stroke. The value of stroke specialist OTs, as compared to non-specialists, is demonstrated by the greater proportion of stroke specialists reporting use of specific assessments and treatments for visual problems.

The most frequently reported treatments delivered by the OTs were ADL training and environmental modification. This is perhaps unsurprising as these are key components of OTs expertise, and these interventions are likely to address the holistic nature of patient problems after stroke and to promote independence and discharge home from hospital. However what is perhaps surprising is that the treatments reported by OTs appear to be the same regardless of whether the patient has visual field problems, visual neglect or eye movement problems. This pattern of delivery of treatment suggests that OTs may not be specifically designing and targeting treatments at a clearly identified visual problem. OTs identified their lack 
of knowledge of the best treatment as a key barrier to management of visual problems, and $70 \%$ of OTs stated that future research should prioritise evidence for the most effective practice/ management/ treatments. This view point is supported by the recently updated SIGN guidelines, which identified the need for high quality research relating to management of visual problems after stroke (13). We believe that future work in this area should prioritise establishing the evidence based for the effectiveness of the range of specific treatment and management strategies for visual impairments following stroke. Currently a series of Cochrane systematic reviews are being carried out to address this need (21-23). Recent data (3) suggest that as many as $68 \%$ of patients with visual problems may have eye movement disorders. Our results demonstrate that only a quarter of OTs report using assessments aimed at identifying eye movement disorders, and only $11 \%$ of OTs report providing treatments for eye movement disorders (although these figures are higher for OTs classing themselves as stroke-specialists). The lack of assessments and treatments used by OTs for eye movement disorders is arguably appropriate, as patients with eye movement disorders ought to be assessed and treated by professionals with specialist knowledge relating to eye movement disorders, such as orthoptists. However only $24 \%$ of respondents reported that they would refer patients with eye movement disorders to an orthoptist, while $53 \%$ reported that they would refer these patients to an ophthalmologist. Further data is required to determine whether patients with eye movement disorders are being identified and referred by other members of the multidisciplinary team, but the results of this questionnaire suggest that OTs may have greater knowledge and understanding of visual field defects and visual neglect than of eye movement disorders. We believe that specialist training for OTs on visual problems should include details of the aetiology, presentation, and management of eye movement disorders. 
Within a stroke inpatient setting there are other health care professionals who may be assessing the visual function of patients. The reported inconsistencies in the use of assessments by OTs, and the apparent failure for certain components of visual function to be assessed (e.g. eye movement) is not therefore necessarily a reflection that the patient has not been assessed. These assessments could be being carried out by another member of the multidisciplinary team. We are currently in the process of carrying out further questionnaires relating to assessment and management of visual problems across Scottish stroke inpatient settings; by both orthoptists and ophthalmologists. The combined responses of these questionnaires will build a more complete picture of the assessments received by patients with stroke in Scotland.

\section{Implications}

The data from this questionnaire study leads us to suggest that; protocols or management plans for visual problems should be introduced within all Scottish stroke inpatient settings; clear referral pathways should be introduced as part of a visual care protocol or management plan; OTs require clear guidance relating to suitable and appropriate assessments of vision after stroke; OT specialist training should include the aetiology and presentation of visual problems after stroke; high quality research into the effectiveness of specific treatments and management strategies should be a priority. Furthermore our data suggest that the assessment and management of eye movement problems may be neglected; we recommend that particular attention should be paid to ensuring that health professionals recognise and understand common eye movement problems after stroke.

Although these data are arguably limited by geography, as the sample was restricted to Scotland, UK, we suggest that OT practice and stroke in-patient settings 
throughout other parts of the UK are highly similar and that these implications therefore could be applied throughout the UK. A similar lack of protocols and referral pathways, training and guidance for health professionals working with stroke patients with visual problems, may also be evident in other countries. This would need to be confirmed with further research in the appropriate geographical areas. Identifying current practices throughout the developed world will be an important step in the identification of promising interventions and effective practices, and in the prioritisation of future research in this neglected field. 


\section{$\underline{\text { References }}$}

1. Stroke Information, Chest, Heart and Stroke Scotland. http://www.chss.org.uk/stroke/: accessed 14/1/2011

2. Lawrence E, Coshall C, Dundas R. Estimates of the prevalence of acute stroke impairments and disability in a multiethnic population. Stroke 2001; 32 (6): $1279-84$

3. Rowe FJ, VIS Group. Visual impairment following stroke. Do stroke patients require vision assessment? Age and Ageing 2009; 38:188-193

4. Kerkhoff G. Neurovisual rehabilitation: recent developments and future directions. J. Neurol. Neuosurg. Psychiatr 2000; 68: 691-706

5. Zihl J. Rehabilitation of Visual disorders after brain injury. Hove, UK; Psychology Press; 2000.

6. Kalra L, Smith DH, Crome P. Stroke in patients aged over 75 years: outcome and predictors. Postgrad Med J 1993; 69: 33-6.

7. Jehkonen M, Ahonen JP, Dastidar P et al. Visual Neglect as a predictor of functional outcome one year after stroke. Acta Neurol Scand 2000; 101: 195201.

8. Wolter M, Shannon P. Visual deficits following stroke: maximising participation in rehabilitation.. Topics in Stroke Rehabilitation 2006;13(3):12-21.

9. Jones SA, Shinton RA. Improving outcome in stroke patients with visual problems. Age and Ageing 2006;35:560-565.

10. Lotery AJ, Wiggam MI, Jackson AJ, Silvestri G, Refson K, Fullerton KJ, Gilmore $\mathrm{DH}$, Beringer TRO. Correctable visual impairment in stroke rehabilitation patients. Age and Aging 2000;29:221-222. 
11. Bouwmeester L.The effect of visual training for patients with visual field defects due to brain damage: a systematic review. J Neurol Neurosurg Psychiatry 2007; 78: 555-564.

12. Royal College of Physicians Intercollegiate Stroke Working Party. National clinical guidelines for stroke. Third edition. July 2008. ISBN: 9781860163340

13. SIGN: Scottish Intercollegiate Guidelines Network. Management of patients with stroke: Rehabilitation, prevention and management of complications, and discharge planning. A national clinical guideline (118). June 2010. ISBN 9781 905813636

14. Lubbock, G. Stroke Care: an interdisciplinary approach. London: Faber; 1983

15. Gillen G, Burkhardt A. Stroke Rehabilitation. A Function-Based Approach. London: Mosby; 2004.

16. Crepeau EB, Cohn ES, Boyt Schell BA. Occupational Therapy. $11^{\text {th }}$ ed. Baltimore: Lippincott Williams and Williams; 2009.

17. Rowland TJ, Cooke DM, Gustafsson LA. Role of occupational therapy after stroke. Ann Indian Acad Neurol 2008;11:99-107.

18. Edwards PJ, Roberts I, Clarke MJ, DiGuiseppi C, Wentz R, Kwan I, Cooper R, Felix LM, Pratap S. Methods to increase response to postal and electronic questionnaires. Cochrane Database of Systematic Reviews 2009, Issue 3. Art. No.: MR000008. DOI: 10.1002/14651858.MR000008.pub4

19. Jamieson K, Brady M, Hagen S, Langhorne P, Capewell A, Bugge C, McClurg D, Chalmers C. A survey of urinary continence care practices in Scottish Stroke Care Settings. Clinical Rehabilitation 2009; 23:762-763.

20. Talbot A, Brady M, Furlanetto DLC, Frenkel H, Williams BO. Oral care and Stroke Units. Gerodontology 2005; 22: 77-8 
21. Pollock A, Hazelton C, Henderson CA, Angilley J, Dhillon B, Langhorne P, Livingstone K, Munro FA, Orr H, Rowe FJ, Shahani U. Interventions for visual field defects in patients with stroke (Protocol). Cochrane Database of Systematic Reviews 2010, Issue 3. Art. No.: CD008388. DOI:

10.1002/14651858.CD008388.

22. Pollock A, Hazelton C, Henderson CA, Angilley J, Dhillon B, Langhorne P, Livingstone K, Munro FA, Orr H, Rowe FJ, Shahani U. Interventions for disorders of eye movement in patients with stroke (Protocol). Cochrane Database of Systematic Reviews 2010, Issue 3. Art. No.: CD008389. DOI: 10.1002/14651858.CD008389.

23. Pollock A, Hazelton C, Henderson CA, Angilley J, Dhillon B, Langhorne P, Livingstone K, Munro FA, Orr H, Rowe FJ, Shahani U. Interventions for agerelated visual problems in patients with stroke (Protocol). Cochrane Database of Systematic Reviews 2010, Issue 3. Art. No.: CD008390. DOI: 10.1002/14651858.CD008390. 


\begin{tabular}{|c|c|c|c|}
\hline & $\begin{array}{l}\text { "I use this } \\
\text { assessment } \\
\text { with every } \\
\text { patient or } \\
\text { regularly" }\end{array}$ & $\begin{array}{l}\text { "I rarely or } \\
\text { never used } \\
\text { this } \\
\text { assessment" }\end{array}$ & $\begin{array}{c}\text { no } \\
\text { response }\end{array}$ \\
\hline Visual Acuity - Distance & $19(34 \%)$ & $23(42 \%)$ & $13(24 \%)$ \\
\hline Visual Acuity - Near & $22(40 \%)$ & $18(33 \%)$ & $15(27 \%)$ \\
\hline Visual Fields (confrontation) & $37(67 \%)$ & $10(18 \%)$ & $8(15 \%)$ \\
\hline Contrast Sensitivity & $5(9 \%)$ & $30(55 \%)$ & $20(36 \%)$ \\
\hline Ocular Movements & $14(25 \%)$ & $27(50 \%)$ & $14(25 \%)$ \\
\hline Binocular Function & $16(29 \%)$ & $23(42 \%)$ & $16(29 \%)$ \\
\hline Visual Attention & $49(89 \%)$ & $6(11 \%)$ & $0(0 \%)$ \\
\hline Visual Scanning & $49(89 \%)$ & $5(9 \%)$ & $1(2 \%)$ \\
\hline Pattern Recognition & $29(53 \%)$ & $15(27 \%)$ & $11(20 \%)$ \\
\hline Visual Memory & $31(56 \%)$ & $16(29 \%)$ & $8(15 \%)$ \\
\hline Visual Tracking & $36(66 \%)$ & $10(18 \%)$ & $9(16 \%)$ \\
\hline Behavioural Inattention Test & $28(51 \%)$ & $23(42 \%)$ & $4(7 \%)$ \\
\hline Brain Injury Visual Assessment Battery & $1(2 \%)$ & $34(62 \%)$ & $20(36 \%)$ \\
\hline Biometrics e-link Evaluation & $11(20 \%)$ & $32(58 \%)$ & $12(22 \%)$ \\
\hline Motor Free Visual Perception Test & $1(2 \%)$ & $35(64 \%)$ & $19(34 \%)$ \\
\hline
\end{tabular}

Table 1. Visual Assessments and Tests; number (and percentage) of OTs reporting use of specific assessments and tests. 


\begin{tabular}{|c|c|c|c|c|}
\hline & $\begin{array}{l}\text { Visual } \\
\text { field } \\
\text { problem }\end{array}$ & $\begin{array}{l}\text { Visual } \\
\text { neglect }\end{array}$ & $\begin{array}{c}\text { Eye } \\
\text { movement } \\
\text { problem }\end{array}$ & $\begin{array}{c}\text { Problem } \\
\text { relating to } \\
\text { spectacle } \\
\text { use }\end{array}$ \\
\hline Refer to ophthalmologist & $25(45 \%)$ & $6(11 \%)$ & $29(53 \%)$ & $4(7 \%)$ \\
\hline Refer to optician & $6(11 \%)$ & $0(0 \%)$ & $3(5 \%)$ & $43(78 \%)$ \\
\hline Refer to orthoptist & $14(25 \%)$ & $8(15 \%)$ & $13(24 \%)$ & $1(2 \%)$ \\
\hline Refer to hosptial low vision service & $2(4 \%)$ & $1(2 \%)$ & $0(0 \%)$ & $0(0 \%)$ \\
\hline Refer to community low vision service & $6(11 \%)$ & $4(7 \%)$ & $2(4 \%)$ & $1(2 \%)$ \\
\hline Refer to psychologist & $1(2 \%)$ & $1(2 \%)$ & $0(0 \%)$ & $(0 \%)$ \\
\hline Treatment delivered by OT & $38(69 \%)$ & $45(82 \%)$ & $6(11 \%)$ & $2(4 \%)$ \\
\hline MDT management plan & $26(47 \%)$ & $29(53 \%)$ & $9(16 \%)$ & $6(11 \%)$ \\
\hline
\end{tabular}

Table 2. Respondents reporting specific referrals/management as the 'likely outcome' for patients with particular visual problems. (NB. Respondents were invited to select as many 'likely outcomes' for each visual problem as they felt relevant. The figures reported are the number (and percentage) of respondents, out of the total of 55 respondents, who ticked each individual referral/management outcome. Many respondents selected multiple outcomes). 


\begin{tabular}{|c|c|c|c|c|c|c|c|c|}
\hline & 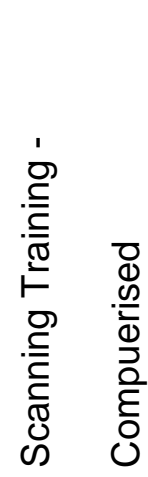 & 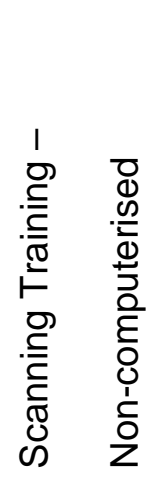 & 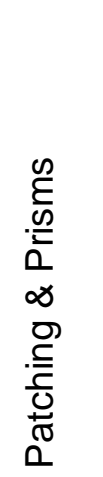 & 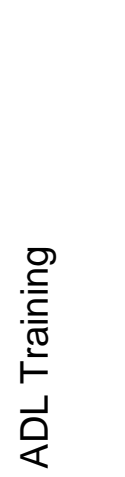 & 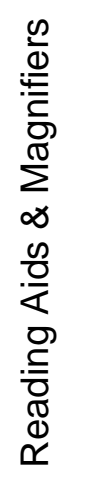 & $\begin{array}{l}\frac{0}{\equiv} \\
\frac{0}{0} \\
\frac{0}{2} \\
\frac{0}{0}\end{array}$ & 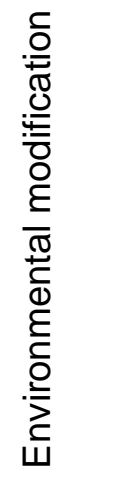 & 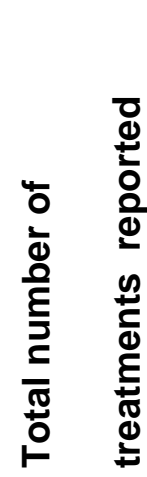 \\
\hline $\begin{array}{l}\text { Visual } \\
\text { Field }\end{array}$ & $\begin{array}{l}23 \\
(11 \%)\end{array}$ & $\begin{array}{l}42 \\
(20 \%)\end{array}$ & $\begin{array}{l}5 \\
(2 \%)\end{array}$ & $\begin{array}{l}49 \\
(23 \%)\end{array}$ & $\begin{array}{l}17 \\
(8 \%)\end{array}$ & $\begin{array}{l}31 \\
(15 \%)\end{array}$ & $\begin{array}{l}45 \\
(21 \%)\end{array}$ & $\begin{array}{l}212 \\
(100 \%)\end{array}$ \\
\hline $\begin{array}{l}\text { Eye } \\
\text { Movement }\end{array}$ & $\begin{array}{c}6 \\
(7 \%)\end{array}$ & $\begin{array}{l}9 \\
(23 \%)\end{array}$ & $\begin{array}{l}2 \\
(2 \%)\end{array}$ & $\begin{array}{l}24 \\
(29 \%)\end{array}$ & $\begin{array}{l}3 \\
(4 \%)\end{array}$ & $\begin{array}{l}13 \\
(15 \%)\end{array}$ & $\begin{array}{l}17 \\
(20 \%)\end{array}$ & $\begin{array}{l}84 \\
(100 \%)\end{array}$ \\
\hline $\begin{array}{l}\text { Visual } \\
\text { Neglect }\end{array}$ & $\begin{array}{c}21 \\
(10 \%)\end{array}$ & $\begin{array}{l}41 \\
(20 \%)\end{array}$ & $\begin{array}{l}1 \\
(1 \%)\end{array}$ & $\begin{array}{l}50 \\
(25 \%)\end{array}$ & $\begin{array}{l}8 \\
(4 \%)\end{array}$ & $\begin{array}{l}34 \\
(17 \%)\end{array}$ & $\begin{array}{l}46 \\
(23 \%)\end{array}$ & $\begin{array}{l}201 \\
(100 \%)\end{array}$ \\
\hline
\end{tabular}

Table 3. Specific treatment interventions: number of OTs reporting use of specific treatments for patients with the different categories of visual problems (and percentage of total number of treatments reported for that visual problem).

(NB. Respondents were invited to select as many treatments for each visual problem as they felt relevant. Many respondents selected multiple treatments). 
Potential stroke in-patient settings in Scotland.

(identified from previous project)

$$
N=71
$$

Researcher telephones each potential setting \& identifies name of OT responsible for providing care in that setting.

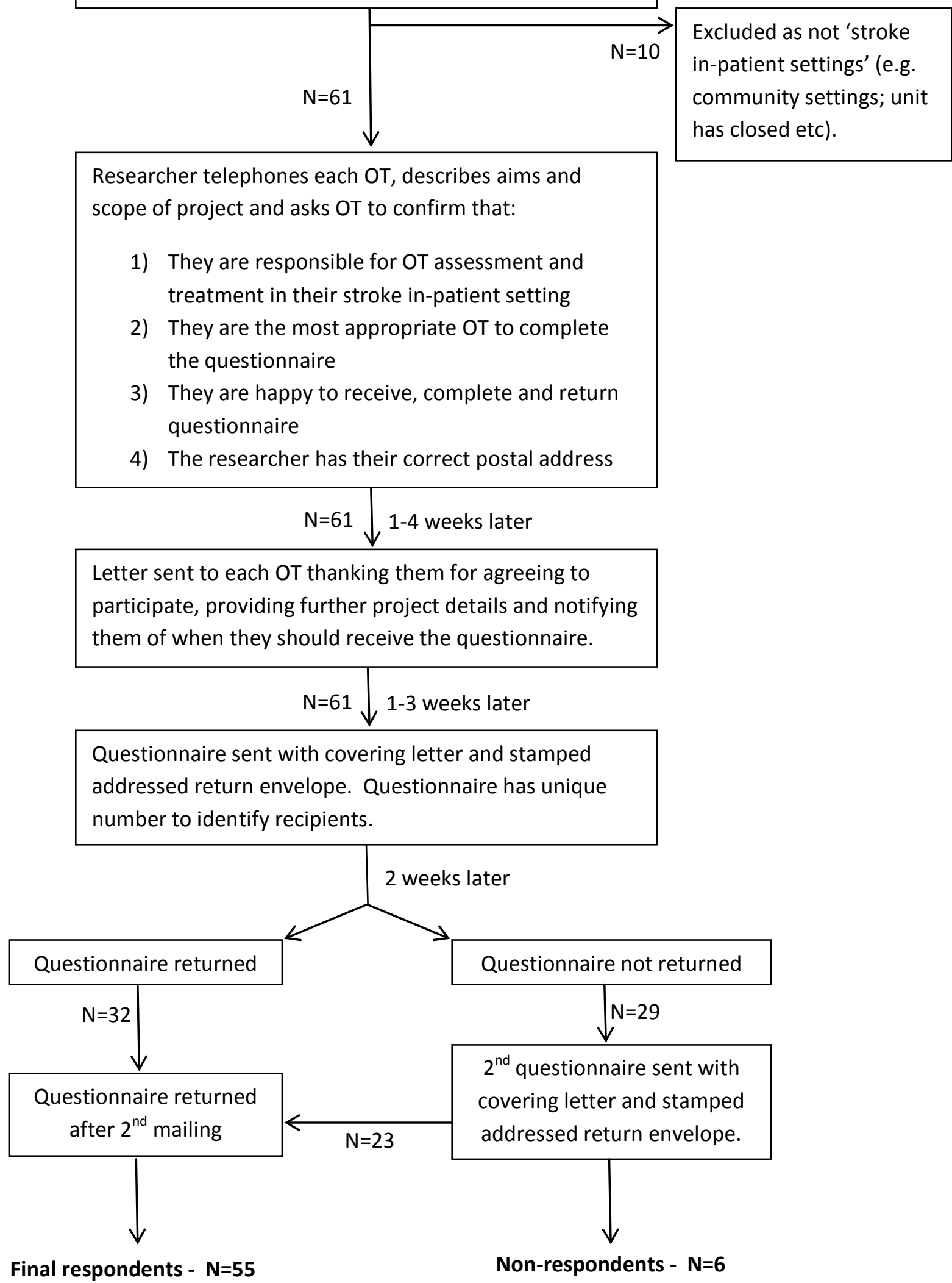




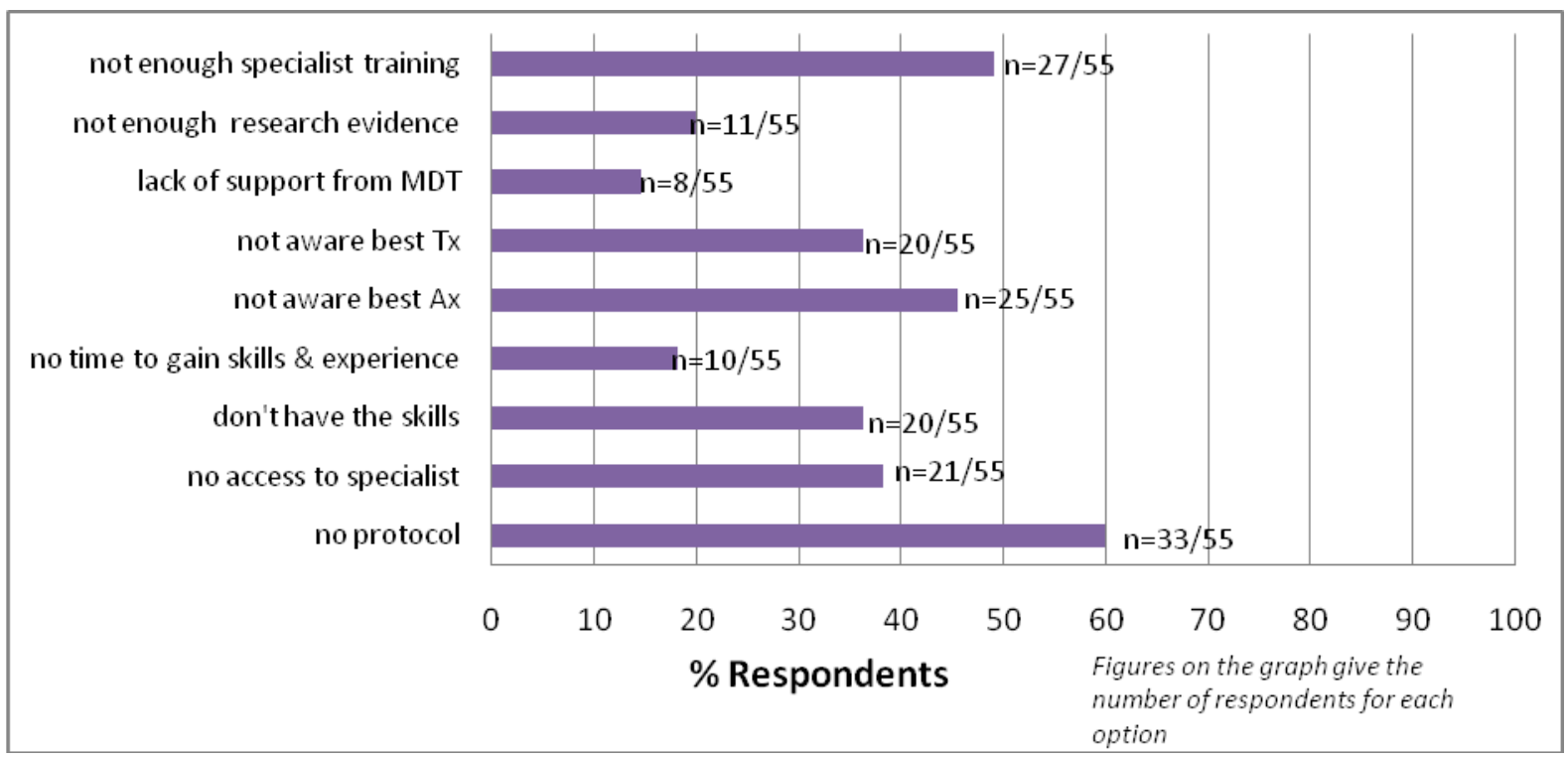

Figure 2. Barriers to the management of visual problems

MDT: Multidisciplinary team; Tx: treatments; Ax: assessments. 